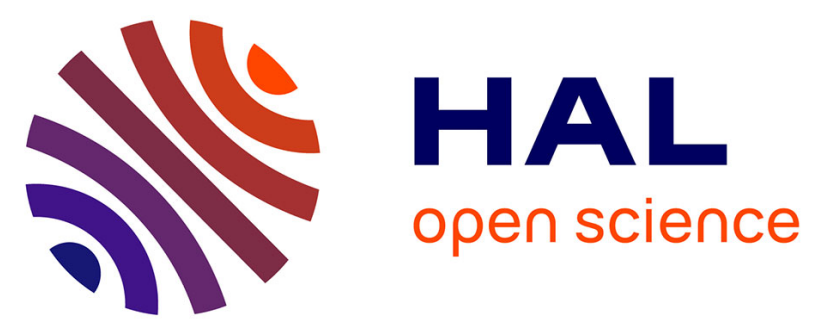

\title{
Modeling contamination at molecular level from elementary tests processing to flight contamination assessment
}

Emilie Vanhove, Vincent Mouysset, Eudes Grosjean, Jean-Francois Roussel, Delphine Faye, Guillaume Rioland

\section{To cite this version:}

Emilie Vanhove, Vincent Mouysset, Eudes Grosjean, Jean-Francois Roussel, Delphine Faye, et al.. Modeling contamination at molecular level from elementary tests processing to flight contamination assessment. Systems Contamination: Prediction, Control, and Performance 2020, Aug 2020, Online Only, United States. pp.16, 10.1117/12.2569120 . hal-03224530

\section{HAL Id: hal-03224530 \\ https://hal.science/hal-03224530}

Submitted on 9 Jun 2021

HAL is a multi-disciplinary open access archive for the deposit and dissemination of scientific research documents, whether they are published or not. The documents may come from teaching and research institutions in France or abroad, or from public or private research centers.
L'archive ouverte pluridisciplinaire HAL, est destinée au dépôt et à la diffusion de documents scientifiques de niveau recherche, publiés ou non, émanant des établissements d'enseignement et de recherche français ou étrangers, des laboratoires publics ou privés. 


\title{
Modeling contamination at molecular level from elementary tests processing to flight contamination assessment
}

\author{
E. Vanhove ${ }^{\mathrm{a}}$, V. Mouysset ${ }^{\mathrm{a}}$, E. Grosjean ${ }^{\mathrm{a}}$, J.-F. Roussel ${ }^{* a}$, D. Faye ${ }^{\mathrm{b}}$, G. Rioland ${ }^{\mathrm{b}}$ \\ ${ }^{a}$ ONERA, The French Aerospace Lab, 2. Av E Belin, 31055 Toulouse, France; ${ }^{b}$ CNES, 18 Av. E \\ Belin, 31400 Toulouse, France
}

\begin{abstract}
The numerical assessment of in-flight contamination is a global process, which needs consistent numerical processing of elementary ground tests and global modeling of in-orbit situations. If the traditional physical approach of Europe is followed, both sides must make use of consistent physical models, and upgrade them consistently. This article presents recent progress performed at ONERA, in collaboration with CNES, in this respect.

For this physical approach, elementary material outgassing tests aim at characterizing each chemical species independently, based on TGA / MS coupling for in situ characterization. Processing large data sets of mass peaks versus time, and interpreting them as resulting from a few outgassed chemical species, each one with its own mass spectrum, requires heavy computations and smart algorithms. The first results shown here are very promising. QCM and mass spec data acquired during TGAs where fitted with very convincing models for deposit reemission and mass spectra for the reemitted species that were identified with database spectra. This makes us confident in the next step consisting in similarly interpreting outgassing QCM + MS measurements in term of discriminated species, although they are all outgassed simultaneously in that case.
\end{abstract}

Keywords: Contamination, chemical species, spacecraft, thermogravimetric analysis, outgassing, desorption

\section{INTRODUCTION}

Contamination analysis aims at assessing and reducing the impact of contamination on flight mission, keeping it under requirement levels. Ground experiments cannot directly lead to such assessments. Transport from contaminant sources to sensitive surfaces is difficult to experimentally test on ground due to chamber effects and is usually estimated through numerical simulations. The extrapolation of necessarily shorter experiments to mission time scales is another challenge. It can either be addressed by direct empirical extrapolation of experimental measurements (of dynamical elementary outgassing tests, such as the US ASTM-1559), or through the physical modeling of these processes and by applying these models to mission flight conditions (an approach more widespread in Europe based on VBQC-type tests). This approach offers more flexibility, by allowing predicting outgassing and even reemission for any temperature profile in flight, such as thermal cycling. However, its representativeness of reality relies on physical assumptions that still need to be confirmed.

Most if not all quantitative measurements of contamination until now were total mass measurements, performed on QCM (Quartz Crystal Microbalances) or traditional balances. These measurements are global, in the sense that they account for the total mass, deposited or outgassed, of all the chemical species stemming from a material. Extrapolating such 7-day isothermal ASTM-1559 data to a 15-year mission through an arbitrary power-law curve, or fitting 5-day temperature-step VBQC data through the sum of unjustified laws for 6 species is equally unsatisfactory. We have been convinced for many year now that the only way forward to really improve this state of the art is to perform a characterization at chemical species level, separating the contribution of each species to outgassing or deposit ${ }^{1,2,3}$. Whereas a total mass can easily be fitted by the sum of several laws regardless of their physical representativeness (exponentials for desorption or diffusion laws can usually equally do the job), a long-hoped-for independent measurement of each chemical species can probably only be discriminatorily fitted by the real physical law, the only one promising an accurate mission time extrapolation. After summarizing some older results showing more accurately why we really need species separation (section 2), this paper reports recent progress on this line of work (section 3).

* Jean-Francois.Roussel@onera.fr; phone +33 562252735 


\section{WHY SPECIES SEPARATION AND DETERMINATION IS NEEDED}

As stated in te introduction, as soon as a physical approach is pursued, a separate characterization of each contaminant species is needed if one does not want to run the risk of ending up with a purely mathematical fit of global data. This is the key to a reliable extrapolation. This is true for outgassing modeling, for reemission, but also for UV fixation, for which a physical modeling was also developed, which also relies on a realistic species level characterization ${ }^{4}$.

Two past achievements are reported here to illustrate the great interest of this approach. At the stage of more basic research, when investigating the behavior of pure compounds and of their mixtures, we had the surprise to see three TGA reemission peaks from a two species deposit (BPA and TPM, cf. Figure 1). The mass spectrometry, which was easier to interpret in this simpler two-species situation, allowed us to confirm the physics at play. The molecules reemitted during the first peak were TPM, with the expected kinetics for pure TPM, hence consistently with a phase of pure TPM. The third peak was BPA as indicated by the mass spec and the consistent kinetics. The unexpected second peak was shown by MS to be TPM. Its kinetics, intermediate between those of pure TPM and pure BPA, indicated that the volatile TPM was trapped by the less volatile BPA in a second mixed phase, until it is released from this phase, probably when BPATPM affinity is thermally compensated and/or the fluidity of the mixture allows the migration of TPM to the surface of the mixture and its desorption.

This experiment illustrates the interest of species separation/analysis through mass spectrometry. It also shows the existence of complex mixture effects in some situations. Fortunately other experiments with realistic contaminant mixtures stemming from real materials exhibited no such effects, which gives us some hope that such complex situation do not arise too often ${ }^{5}$. If they do yet, TGA-MS characterization of molecular species will greatly help detecting them, and maybe treat them quantitatively.

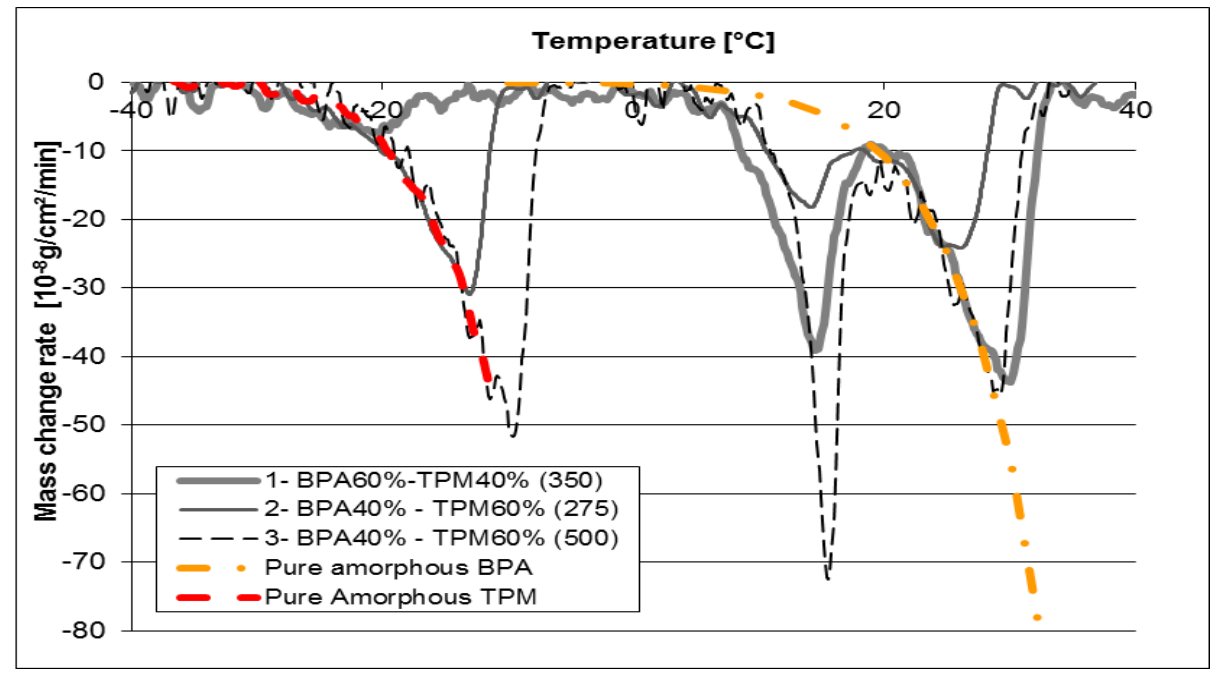

Figure 1. TGA-MS of a 2-contaminant deposit (BPA - Bisphenol A and TPM - Triphenylmethane) exhibiting 3 reemission peaks.

Another very interested achievement, which convinced us to pursue towards species separation, is depicted on Figure 2. Even before developing advanced numerical methods to process MS + QCM data acquired during TGAs (and outgassing) experiments, some simple situations allowed us to reliably discriminate some species. Such was the case for mass peak 91 considered representative of toluene (usually without significant contribution of other species), which we were able to monitor during the outgassing of EC2216 epoxy glue (which was very fortunate, since unlike TGAs outgassing efflux comes as a big mixture of species). In this very specific case we were able to match these data with a modeling of outgassing through a diffusion-limited model, whereas it was impossible with a desorption-limited outgassing model. This is exactly the physical validation, and quantitative analysis we want to generalize. 


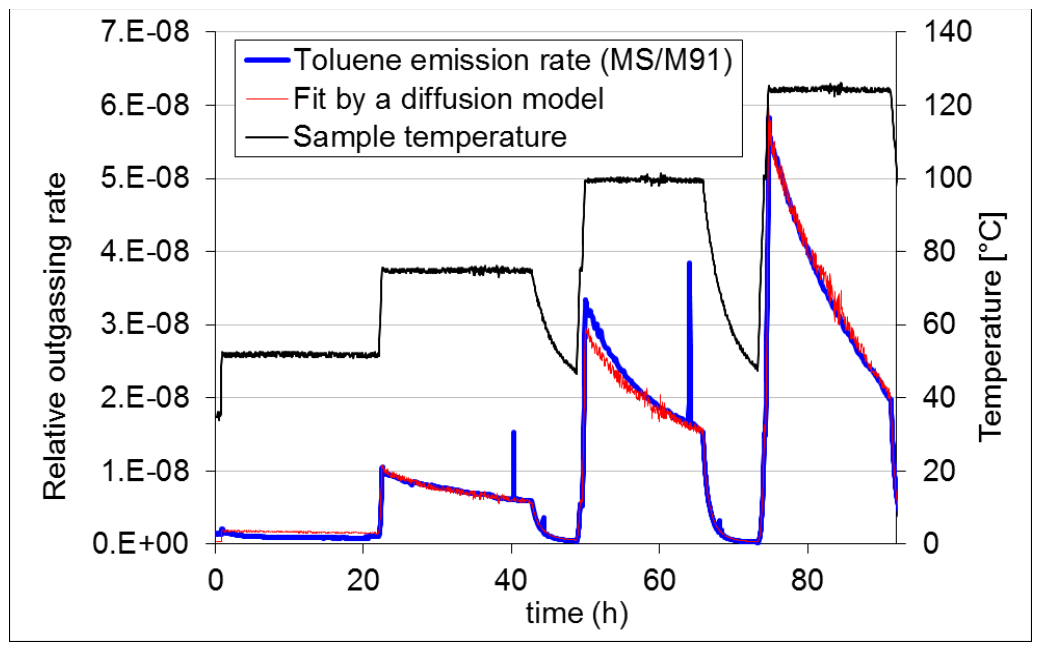

Figure 2. Mass peak 91 measurement (blue) during temperature step outgassing of EC2216 (black), considered to be representative of toluene, compared to a diffusion-limited outgassing model (red).

\section{PROGRESS ON PRACTICAL SPECIES SEPARATION}

These encouraging results pushed us to pursue on the difficult path of species separation. We present here our most recent results in this direction. The overall objective consists in representing (modeling) the QCM and Mass Spectrometer data (all mass peaks versus time) collected during a TGA through a reemission model of a few chemical species, each with a given mass spectrum (the mass peaks generated by each species in the MS). As we shall see the results of this mathematical processing, i.e. the species reemission flux versus time and the mass spectrum of each species, can either be considered completely free/unknown, or (partially) known a priori.

\subsection{First approach}

In a first approach we assumed that each species reemission during the TGA follows a first order law, i.e. a residence time model. It is representative of desorption for sub monolayer deposits. For thicker deposits it introduces some distortion, or can be simply considered as changing of time-function basis (the result can be a linear combination of such functions if one function is ill-suited to physics).

Figure 3 depicts the fit of the total mass reemission from the QCM by ten species each following an order 1 reemission law. In contrast to older approaches the model of course also fits all mass spec data versus time. This huge amount of data is not represented here (see Fig. 7 for an example below). We only give two examples of the mass spectra determined by this processing and their comparison to NIST database spectra in the two following figures. 


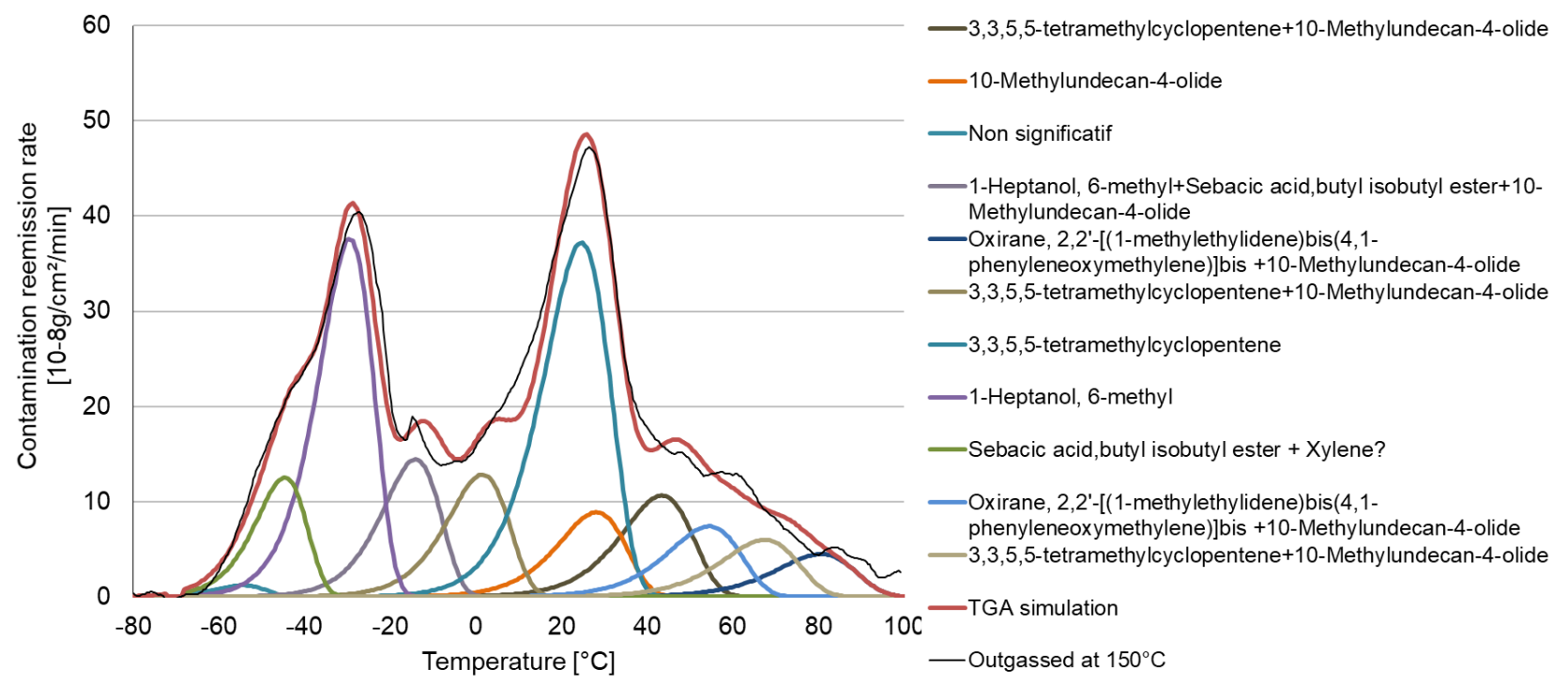

Figure 3. Fit of TQCM and MS data during TGA experiment through the reemission of a 10 species mixture (order 1 law) each with a numerically-determined mass spectrum: plot of the TQCM data compared to the 10 species masses (individual + total).

The numerical and experimental spectra below match quite well, in particular for the heaviest mass peaks (lighter ones are more noisy), which makes us quite confident that we identified the right species. In some cases, data are matched by the sum of two database spectra. If confirmed this fact might indicate the concomitant reemission of two species due to mixture effects similar to those shown in the previous section (lighter species blocked until the heavier is reemitted).

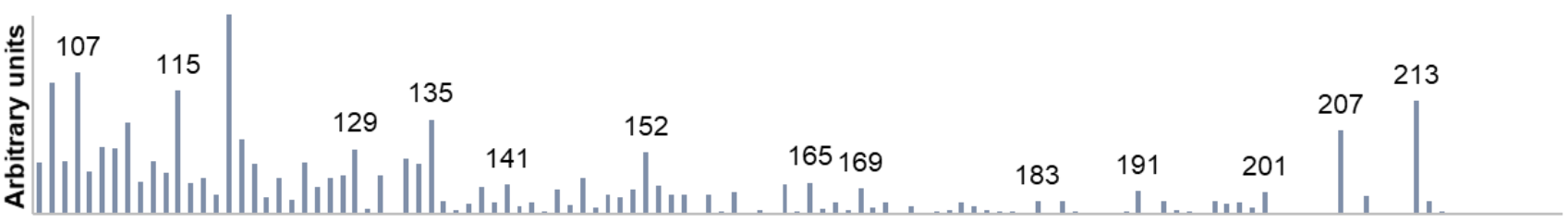

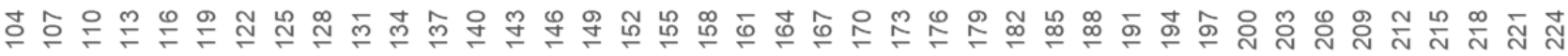

Mass to charge ratios (AMU)

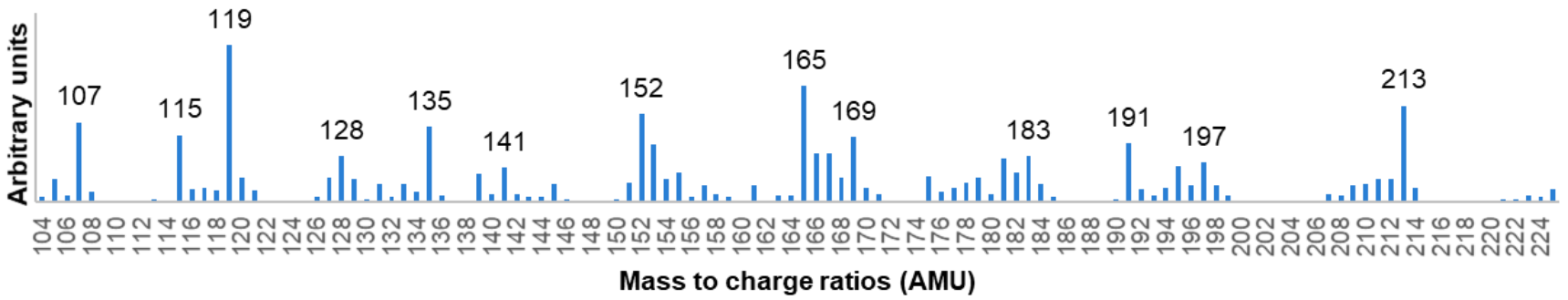

Figure 4. Experimental mass spectrum determined by numerical analysis of mass peak data versus time (upper panel), compared to NIST database spectrum for Oxirane, 2,2'-[(1-methylethylidene)bis(4,1-phenyleneoxymethylene)]bis (lower panel). 

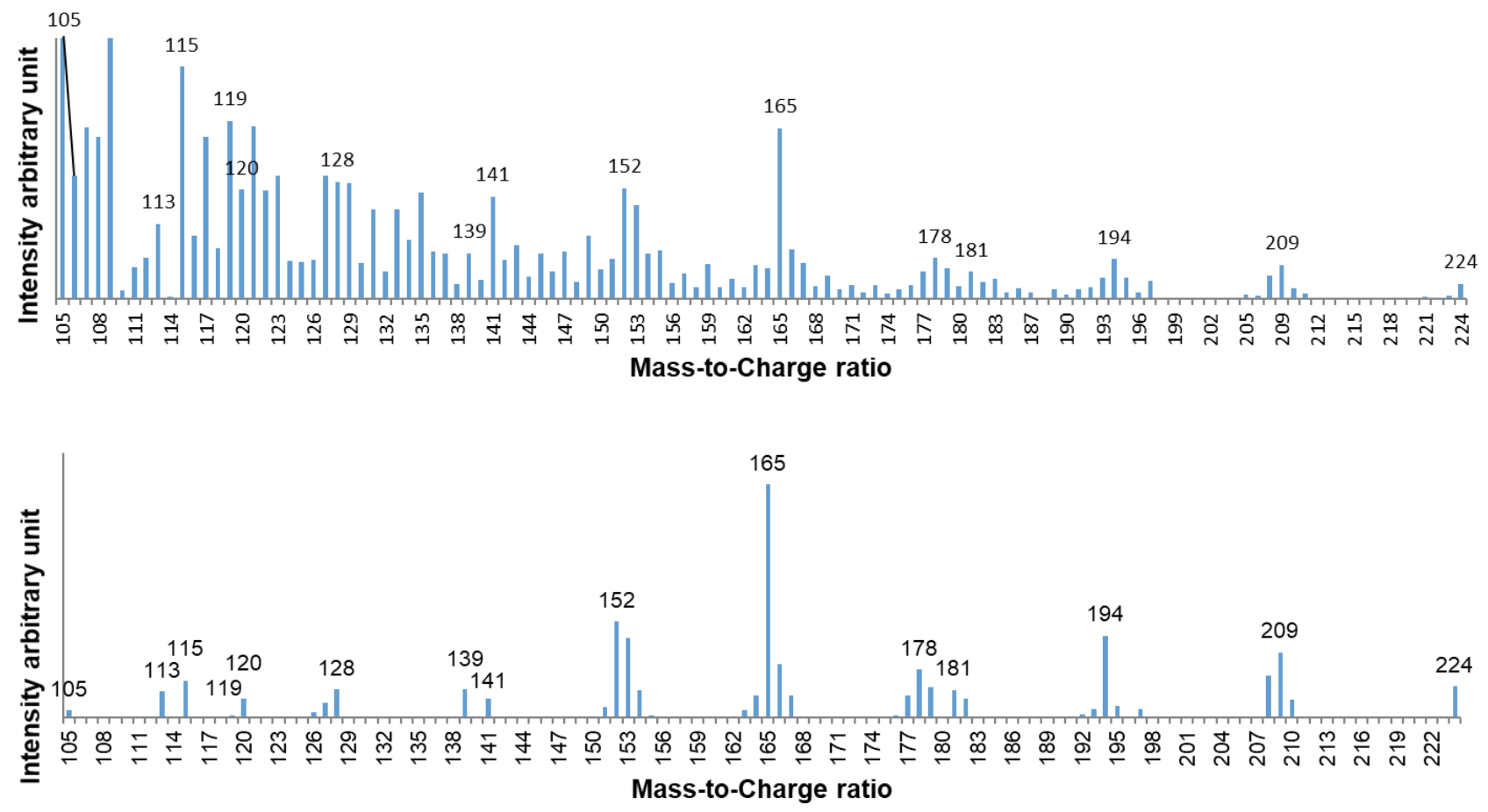

Figure 5. Another experimental mass spectrum determined by numerical analysis (upper panel), compared to NIST database spectrum for Benzene,1,3-dimethoxy-5-[(1E)-2-phenylethenyl] (lower panel).

\subsection{Second approach}

In a second approach we wanted to relax any assumption on the time shape of each species reemission curve (instead of previously assuming it to follow a first law). For modeling the same experiment this way, we were able to keep the mass spectra of the chemical species determined in the first approach, which made the method convergence easier.

The resulting reemission fluxes are represented in Figure 6. The fit of the total mass (black and red curves) is improved although this is not necessarily a sign of improvement of the model (it might just be a reproduction of experimental errors by the model). More interestingly we see that the peaks of each species remain quite similar, i.e. relatively peaked. Some of these peaks even exhibit sharper (steeper) terminations, which might indicated reemission physics closer to an order 0 law (evaporation of thicker deposits) than the order 1 law of the first approach (monolayer desorption).

Figure 7 depicts examples of three mass peaks considered as generated by one species only (10-Methylundecan-4-olide) and the reemitted mass of this species as determined by the code. The similarity of these curves gives a good level of confidence in the fact that these peaks correspond to molecule fragments generated in the mass spec ionization chamber by a single chemical species (or possibly two if they are reemitted concomitantly as conjectured above on some case). 


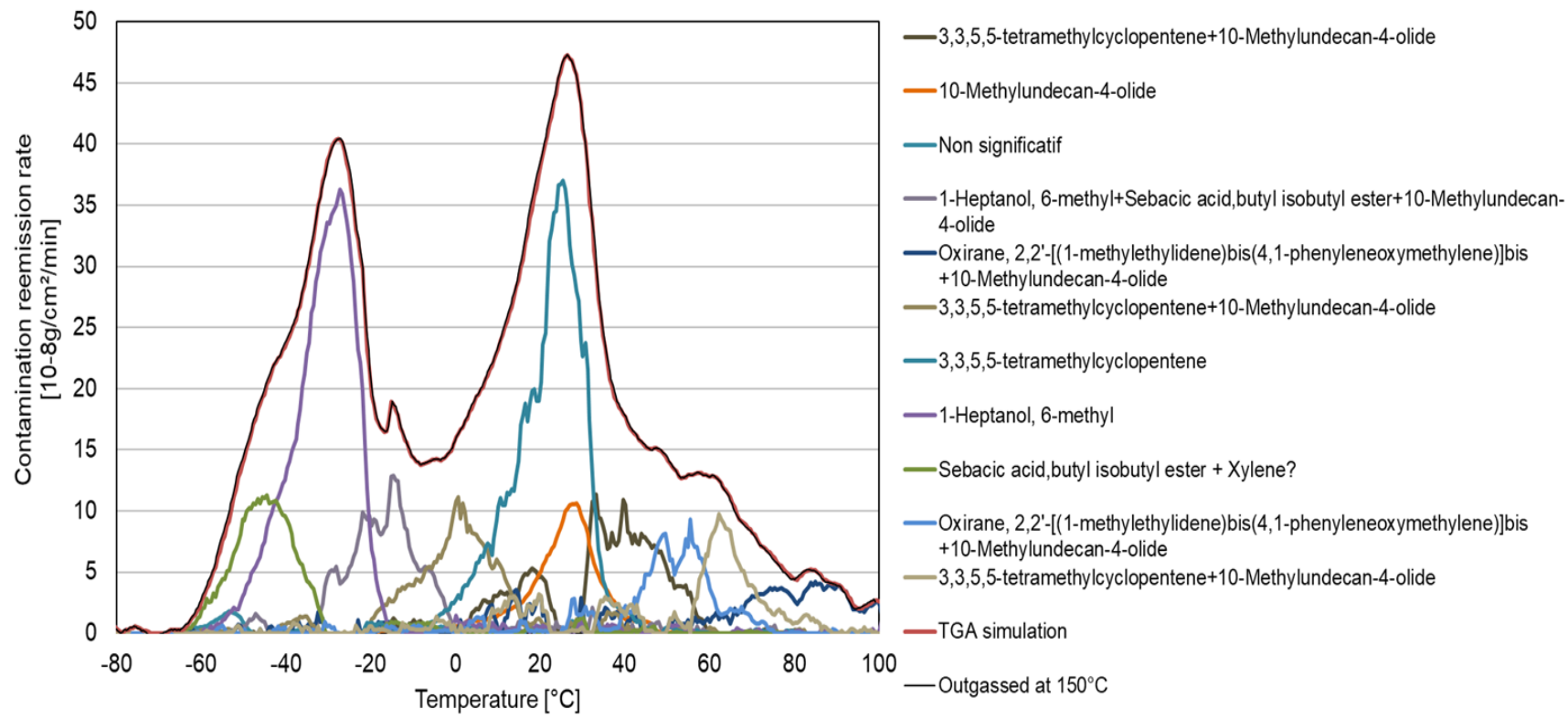

Figure 6. Fit of TQCM and MS data during TGA experiment through the reemission of a 10 species mixture (unconstrained numerically-determined time dependence) each with its previously-determined mass spectrum: plot of the TQCM data compared to the 10 species masses (individual + total).

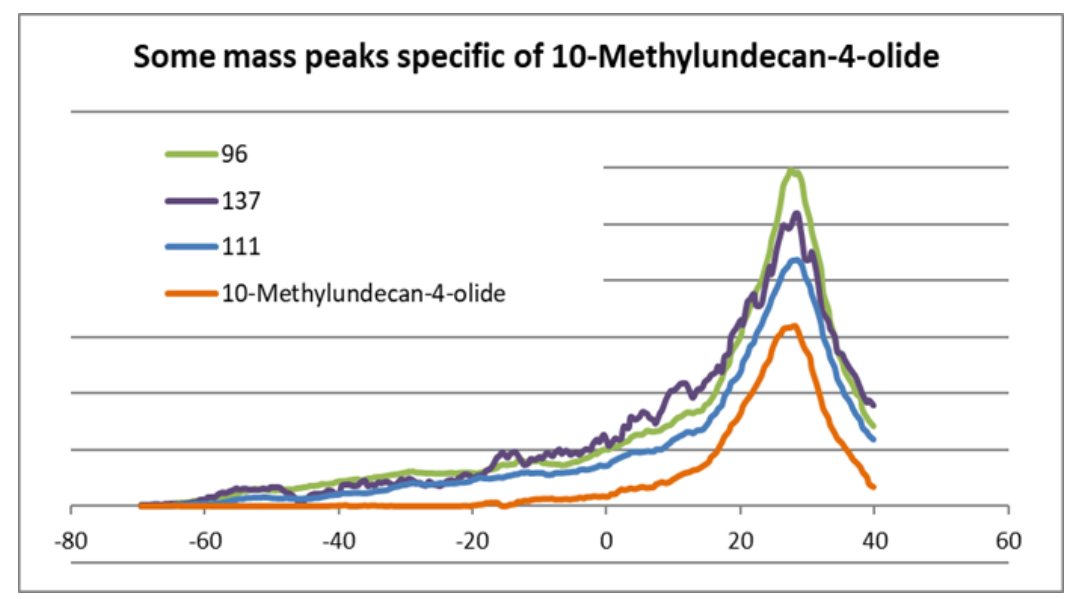

Figure 7. Fit of TQCM and MS data during TGA experiment through the reemission of a 10 species mixture (unconstrained numerically-determined time dependence) each with its previously-determined mass spectrum: plot of 3 mass peaks representative of the species identified as 10-Methylundecan-4-olide.

\section{CONCLUSION AND PERSPECTIVES}

As briefly described in this paper, we made very significant steps on the path of molecule separation and quantitative analysis. TGA data processing still remains to be better analyzed and made more robust, in particular in cases where numerical spectra are matched by the sum of two database spectra.

After improving these TGA analyses, the next big step will be to also fit the outgassing data, again including QCM and mass spec data. This is significantly more complex since all species might be outgassed simultaneously in the absence of 
a pre-separation by the TGA. However using the mass spectra determined during the TGA phase will help analyzing the outgassing phase, as we did in the second approach of the TGA analysis above. This makes us think the mathematical processing of the outgassing continuous MS measurements sold be tractable.

\section{ACKNOWLEDGEMENTS}

The ONERA authors of this paper are grateful to CNES for funding most of the results reported here.

\section{REFERENCES}

[1] Roussel J.-F., Tondu T., Paulmier T. Faye D., van Eesbeek M. and Rampini R., "Progress on the Physical Approach to Molecular Contamination Modeling", Journal of Spacecraft and Rockets, 48 (2), pp. 246-255 (2011).

[2] Vanhove E., Tondu T., Roussel J. F., Faye D. and Guigue P., "In Situ Real-Time Quantitative and Qualitative Monitoring of Molecular Contamination", Journal of Spacecraft and Rockets, Vol. 53, No. 6, pp. 1166-1171 (2016) https://doi.org/10.2514/1.A33505

[3] Roussel J.-F.,Vanhove E.; Grosjean E., Faye D., Rioland G., Rampini R., Ergincan O., "Progress in a physical approach to contamination in Europe", SPIE 10748, Systems Contamination: Prediction, Control, and Performance 2018, 1074809 (Aug. 2018)

[4] Roussel J.-F., Vanhove E., Tondu T., Faye D. and Guigue P. "Ultraviolet Fixation of Molecular Contamination: Physical Model Numerical Implementation and Validation," Journal of Spacecraft and Rockets, Vol. 53, No. 6, pp. 1159-1165 (2016) https://doi.org/10.2514/1.A33504

[5] Tondu T., Vanhove E., Roussel J.-F. and Delphine Faye, "Mixture Effects in Contaminant Reemission", Journal of Spacecraft and Rockets, Vol. 53, No. 6, pp. 1172-1177 (2016) https://doi.org/10.2514/1.A33507 